\title{
Presence of anti-'tissue' transglutaminase antibodies in inflammatory intestinal diseases: an apoptosis-associated event?
}

\author{
Dear Editor,
}

Celiac disease (CD) is described as a permanent intolerance to gluten, characterised by a wide spectrum of lesions in the intestinal mucosa that can ultimately lead to the atrophy of the villi. ${ }^{1}$ Celiac disease is brought about by ingestion of gliadin which triggers the production of $\lg A$ class serum tissue autoantibodies. ${ }^{1}$ Identification of the autoantigen(s) has been the aim of researchers for many years since it has been hypothesised that autoimmune mechanisms are important in the pathogenesis of celiac disease. ${ }^{1-3}$ 'Tissue' transglutaminase (tTG; E.C. 2.3.2.13) or type-Il transglutaminase has been identified as the major auto-antigens of endomysial antibodies found in coeliac disease. ${ }^{3}$ tTG human genes belong to a family of intracellular and extracellular enzymes which catalyse $\mathrm{Ca}^{2+}$-dependent reactions resulting in the post-translational modification of proteins by establishing $\varepsilon(\gamma-$ glutamyl)lysine cross-linkings and/or covalent incorporation of di- and polyamines into proteins. ${ }^{4-6}$ These covalent crosslinks might determine the olygomerization of substrate protein(s) which acquire the peculiar features of resistance to breakage and chemical attack. ${ }^{4-6}$ The tTG gene encodes a protein with a molecular weight of about $80 \mathrm{kDa}^{4-6}$ which is normally expressed in cells localised in peculiar sites within mammalian tissues (endothelial cells, smooth muscle cells and mesangial cells). ${ }^{5}$ TTG is a multifunctional protein which, in its protein cross-linking configuration, plays a complex role in apoptosis. ${ }^{4-7}$ The enzyme has been shown to be induced and activated in apoptotic cells both in vivo and in vitro experimental models. ${ }^{4-7}$ It has been proposed that tTG may play more than one function within the cascade of events that lead to the establishment of the apoptotic phenotype. ${ }^{4,8}$

Dieterich and colleagues ${ }^{3}$ have proposed that ITG is the predominant, if not the only, endomysial autoantigen characteristic for celiac disease. However, as to whether the tTG-induction observed in CD patients represents a primary pathogenic trigger in coeliac disease (CD) is still unknown. In humans, it has been shown that the $C D$ antigens are localised in the extracellular matrix (ECM) and are expressed by fibroblasts. ${ }^{1,9}$ Although tTG is an intracellular enzyme, previous studies have demonstrated the involvement of tTG in diseases characterised by the induction of apoptosis associated with a drastic remodelling of the affected tissue, particularly in ECM. ${ }^{10,11}$ Considering that very little is known on role of cell death in the pathogenesis of degenerative diseases affecting the intestinal mucosa, we have investigated the role of apoptosis in the pathogenesis of CD. To this aim we have studied tTG expression and anti-tTG IgA production in relationship with apoptosis both in CD patients and in other intestinal pathologies, such as Crohn and Ulcerative rectocolitis (UC), showing various levels of mucosal damage and scarring.

Table 1 gives the analysis of anti-tTG IgA levels in sera obtained from patients affected by various intestinal ulcerative diseases as well as from other autoimmune pathologies. CD, Crohn and UC patients showed elevated levels of circulating anti-tTG antibodies which were significantly higher in respect to both healthy individuals and other autoimmune diseases. However, both Crohn and UC patients presented no detectable antiendomysial antibodies (EMA). The lower absolute levels of circulating anti-tTG antibodies detected in Crohn and UC patients compared with those found in CD patients could be due to the minor extent of the lesions. To get direct information on the expression of TTG in CD pathogenesis, we investigated TTG protein localisation by a standard immunohistochemical procedure on serial sections obtained from biopsy samples of the second portion of duodenum from the previously described group of patients. Considering that the enhanced

Table 1 Serum antitransglutaminase IgA OD values and antiendomysial positive results in untreated CD, IBD, ulcer disease, autoimmune diseases and control patients

\begin{tabular}{|c|c|c|c|c|}
\hline Patients & Anti-tTG OD values & $t$ & $P$ & EMA positivity \\
\hline Untreated CD $(n=20)$ & $0.85+0.35$ & 11.638 & $<0.001$ & $20 / 20$ \\
\hline Crohns $(n=26)$ & $0.20 \pm 0.09$ & 5.962 & $<0.001$ & $0 / 26$ \\
\hline UCs $(n=22)$ & $0.18 \pm 0.08$ & 5.264 & $<0.001$ & $0 / 22$ \\
\hline Diabetes $(n=19)$ & $0.11+0.04$ & 1.694 & 0.097 & $0 / 19$ \\
\hline Thyroiditis $(n=10)$ & $0.08 \pm 0.03$ & -0.721 & 0.475 & $0 / 10$ \\
\hline Multiple sclerosis $(n=24)$ & $0.11+0.05$ & 1.618 & 0.112 & $0 / 24$ \\
\hline LESs $(n=15)$ & $0.07 \pm 0.02$ & -1.815 & 0.077 & $0 / 15$ \\
\hline Control patients $(n=29)$ & $0.09 \pm 0.04$ & & & $0 / 29$ \\
\hline
\end{tabular}

For IgA anti-tTG OD values, means and standard deviations are given and $t$-test has been applied. The $t$ and $P$ values reported in the table refer to the statistical test between each population in study versus control patients 

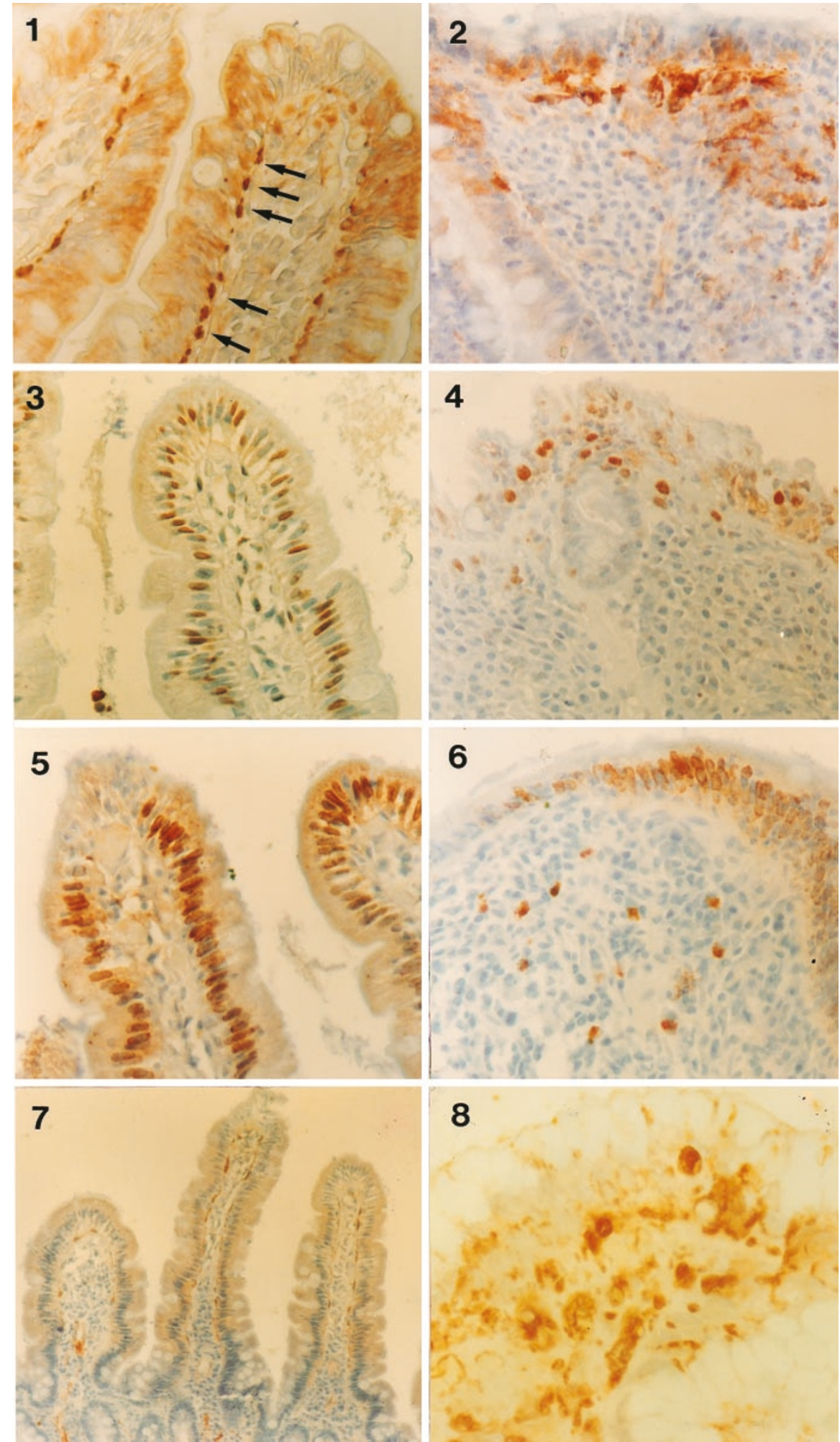

Figure 1 The study was carried out on 20 untreated adult celiac disease patients on gluten containing diet. The diagnosis was based on histological analysis of duodenal biopsies using the eosin-ematoxilin technique. To define mucosal atrophy we measured the villus height/crypt depth ratio. Values $<3: 1$ were considered as index of villus atrophy. As disease control group, we selected 29 patients with gastrointestinal diseases other than CD: 20 patients with gastro-esophageal reflux disease; nine patients with duodenal ulcer disease. All these patients submitted to EGDS with duodenal biopsy for diagnostic purposes, showed a normal mucosa architecture. Sera of 26 patients with Crohn's disease and 22 with ulcerative rectocolitis (UC) were selected as inflammatory chronic intestinal disease control group. As autoimmune disease group, we selected 19 untreated patients with diabetes mellitus, 10 with tyroiditis, 24 with multiple sclerosis and 15 with LES. EMA were detected as previously described. ${ }^{17}$ Anti-tTG antibodies of IgA isotype were measured in by a sandwich type enzyme-immunoassay commercial kit (Eurospital, Italy). ${ }^{18}$ 'Tissue' Transglutaminase, DNA fragmentation, CD95L and $\varepsilon(\gamma$-glutamyl)lysine analysis in the intestinal mucosa from CD patients. Intestinal 
apoptosis is paralleled by the induction of tTG both in physiological and pathological settings, ${ }^{4-7}$ we have analysed whether the lesions occurring in $C D$, as well as in Crohn's diseases (data not shown), are associated with an abnormal onset of apoptosis. In order to correlate the expression of tTG with the induction of apoptosis we have analysed DNA fragmentation in situ by TUNEL and the expression of apoptosis-related genes on consecutive sections from the same patient.

An increased expression of the tTG gene as evidenced by an intense staining with the tTG antibody was detected in all patients in the damaged areas compared to controls (Figure 1). In biopsies obtained from CD patients lesions where the architecture of the mucosa was still preserved, the positivity to the tTG antibody was specifically confined to the enterocytes localised in the upper part of the villi and very limited staining was detected in the submucosa (Figure 1, panel 1). Furthermore, tTG expression was also detected in fibroblasts lining the intestinal epithelium (Figure 1, panel 1). Comparative analysis of serial sections obtained from the same patient (Figure 1, panels 1,3) showed that DNA fragmentation and tTG staining colocalised in post-mitotic enterocytes present in the upper part of the villi; by contrast, no DNA fragmentation was observed in the lamina propria where the tTG-positive fibroblasts were detected. It is interesting to note that the enterocytes showing DNA fragmentation and tTG expression also displayed a strong induction of CD95L (Figure 1, panel 5). CD95L immunostaining were also detected in activated lymphocytes localised in the lamina propria and in the submucosa (Figure 1, panel 5). The comparative analysis of cell morphology, tTG and CD95L expression and TUNEL staining demonstrates that apoptosis occurs in early stages of the diseases and primarily affects the enterocytes. In fact, the tTG-positive fibroblasts localised in the submucosa do not show the biochemical and morphological features of cells undergoing apoptosis (Figure 1).

In the CD lesions with a complete flattening of the mucosa a different expression pattern was observed. The positivity to the tTG antibody was detected not only in cells, but predominantly in the ECM (Figure 1, panel 2). In the same lesions, very few cells were found positive to both CD95L and TUNEL staining, thus indicating that the enterocyte undergoing apoptosis in the earlier stages of the disease were probably already lost in the intestinal lumen. By contrast, the tTG-positive cells detected in the lesions appeared to be preferentially localised in the scarring tissue in proximity of the gut lumen where these cells were releasing their contents into the ECM (Figure 1, panel 2). In the wound healing area close to the intestinal lumen, we also detected an intense staining with a specific antibody recognising the $\varepsilon\left(\gamma\right.$-glutamyl)lysine cross-link, ${ }^{11}$ thus indicating that the released enzyme was actively crosslinking proteins localised in the ECM (Figure 1, panel 8).

The data reported here indicate that the presence of anti-tTG antibodies is not a specific event occurring in CD patients, but is a general phenomenon related to mucosal lesions rather than to the autoimmune nature of $C D$. In keeping with this hypothesis we detected both circulating anti-tTG and induction of apoptosis (data not shown) in other pathologies leading to intestinal lesions such as Crohn and UC, but not in other specific and systemic autoimmune diseases (diabetes, thyroiditis, multiple sclerosis and LES). We would like to hypothesise that the accumulation of tTG in the enterocytes as well as its release in the ECM are a consequences of the induction of apoptosis in those regions undergoing the destruction typical of severe CD-associated lesions. In fact, the enterocytes become tTG-positive and show the morphological and biochemical features typical of apoptotic cells including the expression of the CD95L in early stages of the diseases. Noteworthy, in CD patients showing a complete flattening of the mucosa, a large proportion of the TTG staining was detected in the ECM of the lesion.

Although defects in the CD95 pathway have been shown to play an important role in several autoimmune diseases, ${ }^{8,12}$ very little is known about the CD95/CD95L pathway in CD. Here we present data suggesting the involvement of this receptor in the $C D$ pathogenesis. Interestingly, a deregulated tTG is present in autoimmunity-prone MRL Ipr/lpr mice, ${ }^{13}$ where tTG is also detected into the ECM similarly to the wound healing process and tissue repair occurring in CD lesions. Several studies have indicated that tTG, by crosslinking a number of proteins such as collagens, fibronectin, laminin, nidogen and Transforming Growth Factor-1, might play an important role in the modification of the ECM occurring in degenerative diseases. ${ }^{11,14}$ The presence of the tTG in the ECM in CD lesions might represent per se an important pathogenic event. In fact, displaying of cryptic molecular determinants has been proposed to play an important role in the induction of the pathogenic autoimmune response. ${ }^{8}$ The question arises as to how the activation of the tTG released in the ECM may lead to the formation of pathogenic neo-determinants. ${ }^{8}$ As evidenced by the presence of high levels of $\varepsilon(\gamma$-glutamyl)lysine crosslinks

mucosae from CD patients were biopsied, fixed and stained with anti-tTG $(1-2,7)$ anti-CD95L $(5,6)$, or anti- $\varepsilon(\gamma$-glutamyl)lysine crosslinks $(8)$ antibodies as well as by TUNEL for DNA fragmentation $(3,4)$ as previously described. ${ }^{10,12,13}$ Compared to controls $(7)$ in early stages of the disease $(1,3,5)$ tTG staining is detected in the enterocytes localised in the upper part of the villi and in the fibroblasts lining the intestinal epithelium (arrows; 1 ), while DNA fragmentation (3) and a strong CD95L staining (5) were confined to the enterocytes. In the cute stage of the disease $(2,4,6,8)$, characterized by villus flattening, a large proportion of the tTG staining was detected in the extracellular matrix of the lesion and only a limited number of fibroblasts were stained (2). In the same lesions, very few cells were found positive to both TUNEL (4) and CD95L (6) staining. Note the intense staining detected by both the TTG (2) and anti- $\varepsilon(\gamma$-glutamyl)lysine crosslink antibodies (8) in ECM of the intestinal areas showing a complete flattening of the mucosa. The intense staining detected with the anti- $\varepsilon(\gamma$-glutamyl)lysine crosslinks antibody in the ECM (8) indicate that the released TTG is active 
compared to controls (data not shown), the extracellular tTG localised in CD lesions is active. Hence, it is very likely that the tTG-mediated polymerisation of ECM proteins may generate new self-antigens and thus contributing to elicit the autoimmune responses. In keeping with this assumption, the binding of gliadin to reticular matrix components is $\mathrm{Ca}^{2+}$-dependent, is inhibited by putrescine, and by preincubation with antibodies against tTG suggesting that binding of gliadin might be a tTG-mediated event. ${ }^{15,16}$ Taken together these findings suggest an important complex role for tTG and apoptosis in the pathogenesis of $C D$.

\section{Acknowledgements}

This work was supported by grants from 'AIDS', Ricerca Corrente and Finalizzata Projects from the Ministero Sanita and UE.

MG Farrace ${ }^{1}$, A Picarelli², M Di Tola², L Sabbatella², OP Marchione ${ }^{3}$, G Ippolito ${ }^{4}$ and M Piacentini, ${ }^{*, 1,4}$

1 Department of Biology, University of Rome, "Tor Vergata", Italy;

2 Department of Clinical Science, University of Rome 'La Sapienza', Italy;

3 Instituto 'Proda', Roma, Italy

4 IRCCS 'L. Spallanzani', Rome, Italy

* Corresponding author: M Piacentini, Dipartimento di Biologia, Università di Roma 'Tor Vergata', Via della Ricerca Scientifica, 00133 Rome, Italy.

Tel: 396-72594370; Fax: 396-2023500;

E. mail: MAURO.PIACENTINI@UNIROMA2.IT
1. Maki M et al (1997) Lancet 349: 1755-1759

2. Ferreira $\mathrm{M}$ et al (1992) Gut 33: $1633-1637$

3. Dieterich W et al (1997) Nature Med 7: 797-801

4. Melino G et al (1998) FEBS Lett. 430: 59-63

5. Fesus L et al (1991) Eur. J. Cell Biol. 56: 170-177

6. Piacentini M (1995) Curr. Top. Microbiol. 200: 163-176

7. Fesus L et al (1989) FEBS Lett. 245: 150-154

8. Colizzi V et al (1999) Immunol. Today 20: 134-138

9. Marttinen A et al (1997) Eur. J. Clin. Invest. 27: 135-140

10. Piacentini M et al (1999) J. Pathol. 189(1): $92-98$

11. Johnson TS et al (1997) J. Clin. Invest. 90: 2950-2960

12. Thompson CB (1995) Science 267: 1456-1462

13. Piredda $L$ et al (1997) Cell Death Differ. 4: 463-472

14. Mirza A et al (1997) Am. J. Physiol. 272: G281-G288

15. Maki M (1996) Lancet 348: $1046-1047$

16. Uhlig H et al (1998) Autoimmunity 28: 185-195

17. Picarelli A et al (1996) Lancet 348: 1065-1067

18. Picarelli A (2001) J. Intern Med. 249: 181-188 